Research Article

\title{
Nerve Conduction Studies in Patients with Lumbosacral Radiculopathy Caused by Lumbar Intervertebral Disc Herniation
}

\author{
Safa Yousif $\mathbb{D}^{1},{ }^{1}$ Ammar Ahmed, ${ }^{1}$ Ahmed Abdelhai, ${ }^{2}$ and Afraa Musa ${ }^{1}$ \\ ${ }^{1}$ Department of Physiology, Faculty of Medicine, University of Khartoum, Khartoum, Sudan \\ ${ }^{2}$ Department of Orthopaedics Surgery and Traumatology, Faculty of Medicine, University of Khartoum, Khartoum, Sudan \\ Correspondence should be addressed to Safa Yousif; safaayousif27@yahoo.com
}

Received 22 August 2020; Revised 17 October 2020; Accepted 30 October 2020; Published 16 November 2020

Academic Editor: Andreas K. Demetriades

Copyright (c) 2020 Safa Yousif et al. This is an open access article distributed under the Creative Commons Attribution License, which permits unrestricted use, distribution, and reproduction in any medium, provided the original work is properly cited.

Background. Nerve conduction studies (NCS) are electrodiagnostic tests used to evaluate peripheral nerves functions and aid in the assessment of patients with neuromuscular complaints. There is contrasting evidence concerning the use of NCS in the assessment of patients with lumbosacral radiculopathy. Objectives. This study was conducted to evaluate nerve conduction studies abnormalities in patients with lumbosacral radiculopathy and to find out their relation to abnormal physical examination findings. Materials and Methods. Twenty-seven patients with lumbosacral radiculopathy caused by L4/5 or L5/S1 intervertebral disc prolapse confirmed by magnetic resonance imaging (MRI) were recruited in the study. Twenty-five healthy subjects matched in age and sex served as control. Motor nerve conduction study bilaterally for both common peroneal and tibial nerves, F-wave for both nerves, and H-reflex had been conducted. Results. No significant difference was found in the motor nerve conduction study parameters (latency, amplitude, and conduction velocity) between the patients group and the control group. There was significant prolongation in $\mathrm{H}$-reflex latency of both symptomatic and asymptomatic side in the patients group compared to the control group $(P<0.05)$. Also, F-wave latencies ( $\mathrm{F}$ minimum, $\mathrm{F}$ maximum, and $\mathrm{F}$ mean) of the tibial nerve were significantly prolonged $(P<0.05)$ compared to control. Conclusion. Prolonged H-reflex latency was the commonest encountered abnormality in our study followed by F-wave latencies of the tibial nerve.

\section{Introduction}

Back pain in the third and fourth decade of life is frequently caused by lumbar disc herniation. Most people relate their back and leg pain to a traumatic incident as the pain is usually brought on by repetitive twisting, bending, or heavy lifting [1]. $95 \%$ of the lumbar disc herniation involves $\mathrm{L} 4 / 5$ and L5/S1 level, the latter being the commonest [2].

Radiculopathy due to lumbar disc herniation is defined by North American Spine Society as localized displacement of disc material beyond the normal margins of the intervertebral disc space resulting in pain, weakness, or numbness in a myotomal or dermatomal distribution [3].

Lumbar disc herniation is suspected from the history and clinical examination. Plain radiographs can be used to exclude infection, tumors, or other anomalies but are limited in diagnosing lumbar disc herniation or other intraspinal lesions. Magnetic resonance imaging (MRI) is the most useful tool for identifying structural abnormalities in the intervertebral disc area [1]. Nerve conduction studies (NCS) are electrodiagnostic tests (EDX) that assess peripheral nerve functions and can be used to evaluate patients with neuromuscular complaints.

Radiculopathy is a pathological process that affects the nerves at the root level. Patients usually present with sensory symptoms but motor symptoms also can exist either mixed with sensory symptoms or alone. Because the nerve affected is proximal to the dorsal root ganglia, sensory nerve conduction study is usually normal. For assessing patients with lumbosacral radiculopathy, motor studies of the common peroneal nerve and posterior tibial nerve can be performed. Late responses (F-wave and H-reflex) provide information about the proximal nerve segment (nerve root) [4]. 
Studies evaluating the use of nerve conduction studies in the diagnosis and assessment of patients with lumbosacral radiculopathy vary widely [5-15]. Nerve conduction studies have the advantage of being a noninvasive, objective, and reproducible tool to assess the physiologic integrity of the nerve root. NCS are not widely used in assessing patients with lumbosacral radiculopathy. NCS might provide a useful aid in patients' management with regard to options of treatment, postoperative evaluation of recovery, and detection of recurrence. There is a need for more studies to be conducted to correlate the findings of the NCS in patients with lumbosacral radiculopathy with the clinical manifestations of the disease, provided that there is a limited number of published researches addressing this issue.

The objective of our study was to evaluate nerve conduction studies (NCS) abnormalities in patients with lumbosacral radiculopathy and to find out their relation to abnormal physical examination findings.

\section{Materials and Methods}

2.1. Study Design and Sample. This study was a cross-sectional analytic study conducted in the duration from July to November 2016. The sample size was calculated using G-power software. The sampling technique was convenient sampling.

2.2. Participants. Two groups of subjects were studied: patients and control. Twenty-seven patients were selected; their age ranged between 23 and 60 years $(18$ males and 9 females). Patients were selected from Ibrahim Malik Teaching Hospital referred clinic and Ribat University Hospital referred clinic. Also, patients were recruited from specialized clinic in the Royal Scan diagnostic center, Mr. Ahmed Abdelhai specialized clinic.

\subsection{Patients Group}

2.3.1. Inclusion Criteria. Patients with L4/5 and/or L5/S1 intervertebral disc prolapse confirmed by MRI examination in addition to one or more of the following were included in the study: symptoms appropriate with the MRI level of the disc prolapse, motor weakness, sensory impairment, absent ankle jerk, and/or positive straight leg raising test.

2.3.2. Exclusion Criteria. Patients with history suggestive of polyneuropathy (e.g., diabetes), radiculopathy due to other pathology (e.g., tumor, instability), and/or previous spinal surgery were excluded from the study.

2.3.3. Control Group. Twenty-five healthy subjects were selected for the control group. Control group participants were selected from volunteers who work or study in Faculty of Medicine of Khartoum University. The age range of the control group was between 24 and 60 years (15 males and 10 females). The control group was matched in age and gender with the patients group.
Inclusion criteria included subjects with no history of radiculopathy or neuromuscular problems or any risk factors for having impaired nerve function (alcohol abuse, diabetes, renal problem, neurotoxic drugs, and hypothyroidism), and normal neurological examination.

The study was approved by the ethics research committee of the Faculty of Medicine, Khartoum University. Informed written consent was taken from all participants, and their confidentiality was highly conserved.

\subsection{Data Collection}

2.4.1. Clinical Assessment. Clinical assessment was performed by the principal investigator and took place in Physiology Department, Faculty of Medicine, Khartoum University. History taking included duration of symptoms and the limb involved, in addition to the history of weakness or sphincteric disturbance, and the distribution of sensory symptoms. Pain and/or numbness radiating to the lateral malleolus, lateral foot, heel, or plantar surface of the foot was considered to arise from S1 nerve root involvement. If pain or numbness described by the patient radiated to the anterolateral leg, dorsum of the foot, or the great toe, symptoms were attributed to L5 nerve root involvement. Physical examination included the examination of gait, the motor examination of the lower limb (tone, power, ankle reflex), touch and pinprick sensation, and straight leg raising test.

2.4.2. MRI. Patients' lumbosacral MRI was reported by consultant radiologists blinded to the physical examination findings and the reports were used by the principal investigator to address inclusion criteria.

2.4.3. NCS. NCS were performed using the Digital Medelec Synergy machine software version (10.1) and performed by the principal investigator in Physiology Department, Faculty of Medicine, Khartoum University. Both limbs were studied and NCS reviewed by neurophysiologists blinded to the physical examination findings. Tests performed included motor nerve conduction study of the tibial and common peroneal nerves, F-wave latencies of both nerves, and H-reflex latency of the tibial nerve. Also, the sural nerve sensory nerve conduction study was performed to exclude patient with polyneuropathy.

The following is a description of the conducted NCS tests and the machine setting for each test illustrated in Table 1.

\subsubsection{Tibial Nerve Compound Motor Action Potential} (CMAP). A recording electrode was placed over the abductor halluces muscle. Two stimulation sites were used, distal and proximal. The distal stimulation site was placed $10 \mathrm{~cm}$ proximal to the recording electrode, posterior to the medial malleolus. An electrode placed in the middle of the popliteal crease, served as the proximal stimulation site. Distal latency, amplitude, and conduction velocity were the parameters studied. 
TABLE 1: Machine setting for nerve conduction studies [16].

\begin{tabular}{lcccc}
\hline Setting & Sweep velocity $(\mathrm{msec} /$ division $)$ & Sensitivity $(\mu \mathrm{v} /$ division $)$ & Filters high/low $(\mathrm{kHz} / \mathrm{Hz})$ & Stimulator duration/rate $(\mathrm{msec} / \mathrm{Hz})$ \\
\hline Motor NCS & 2 & 5000 & $10 / 2$ & $0.2 / 1$ \\
Sensory NCS & 1 & 10 & $2 / 20$ & $0.1 / 2$ \\
H-reflex & 10 & 500 & $10 / 20$ & $1 / 0.5$ \\
F-wave & 10 & 500 & $10 / 20$ & $0.2 / 1$
\end{tabular}

This table is reproduced from [16] (under the Creative Commons Attribution License/public domain).

2.4.5. Peroneal Nerve CMAP. A recording electrode was placed over the extensor digitorum brevis. Two stimulation sites were used, distal and proximal. The distal stimulation site was in the dorsal lower leg between the tendons of tibialis anterior and extensor hallucis longus $9 \mathrm{~cm}$ proximal to the recording electrode. The proximal stimulation site was about $3-4 \mathrm{~cm}$ distal to the proximal tip of the fibular head. Parameters studied were distal latency, amplitude, and conduction velocity.

2.4.6. F-Wave. F-wave of the common peroneal and tibial nerves was studied. Electrodes placement was similar to the CMAP recording. The distal stimulation site was used and ten subsequent stimuli were delivered. The latencies recorded and studied were the F-minimum, F-maximum, and F-mean.

2.4.7. H-Reflex. The posterior tibial nerve was stimulated in the center of the popliteal crease and recorded over the soleus muscle. The parameter studied was the shortest latency obtained.

2.4.8. Sural Nerve Sensory Nerve Action Potential (SNAP). The recording electrode was placed behind the lateral malleolus, and the stimulating electrode placed in the midcalf $14 \mathrm{~cm}$ proximal to the recording electrode. Parameters recorded were the latency, amplitude, and conduction velocity. Sural nerve SNAP was studied to exclude participants with polyneuropathy.

2.4.9. Data Analysis. Software Statistical Package for the Social Sciences (IBM SPSS) version 20 was used for data analysis. Mean and standard deviation were calculated to describe continuous variables and frequencies were calculated to describe categorical variables. Data obtained from both groups were compared using the independent $t$-test. Parameters of the symptomatic and asymptomatic side were compared using the paired $t$-test. Association between clinical findings and NCS studies abnormalities was carried out by Chi-Square/Fisher's Exact test. $P$ value less than 0.05 was considered significant.

\section{Results}

The total number of patients included in the study was 27 , the mean age was $41.6 \pm 11.7$ years (mean \pm standard deviation), and $66.67 \%$ were male. The number of healthy subjects was 25 , the mean age was $37.24 \pm 10.4$ years, and 60 $\%$ were male.

The mean duration of symptoms was $20.7 \pm 26.9$ months, and $55.6 \%$ had symptoms for more than 6 months. The left limb was involved in $62.96 \%$ of patients, right limb was involved in $29.63 \%$, and only $7.4 \%$ of patients showed bilateral limb involvement. L5 dermatomal distribution of paresthesia was described in $37.04 \%$ of patients, S1 dermatome was involved in 59.26\%, and 3.7\% showed involvement of both dermatomes.

Regarding physical examination, an antalgic gait was detected in 8 patients and listing in 5 patients $(29.6 \%$ and $18.5 \%$, respectively). Ankle jerk was absent in 8 patients (29.6\%), and impaired touch sensation was detected in 15 patients (55.6\%). A positive straight leg raising (SLR) test was the most common encountered abnormality (16 patients, 59.3\%).

Cut-off values obtained from the control group motor nerve conduction studies were used as a reference value to classify patients' values into normal or abnormal (Table 2). CMAP amplitude of the common peroneal nerve was reduced in $9.3 \%$ of the 54 recorded nerves, while velocity was reduced in $3.7 \%$ of nerves. There was no statistically significant difference between the patients group and the control group in the parameters of common peroneal nerve $(P>0.05)$. Tibial nerve CMAP amplitude was reduced in $3.7 \%$ of the 53 recorded nerves, and velocity decreased in $11.1 \%$. Also, no statistically significant difference between the patients group and the control group was obtained $(P>0.05)$.

F-wave minimum, maximum, and mean latencies of the common peroneal nerve were measured in 40 nerves and F-wave was absent in 14 nerves. No statistically significant difference was found between the patients group and the control group $(P>0.05)$. No statistically significant difference was found between symptomatic and asymptomatic side in the patients group. Also, no statistically significant difference was found between symptomatic side in the patients group and the control group, or between the asymptomatic side in the patients group and the control group.

F-wave latencies of the tibial nerve were obtained in 54 nerves, and there was a statistically significant difference between the patients group and the control group in minimum, maximum, and mean latencies. The maximum latency was the most significant $(P<0.05)$ (Table 3$)$. Tibial nerve $\mathrm{F}$-wave latencies of symptomatic and asymptomatic sides, when compared to the control group, revealed a significant difference in the maximum latency (Table 4). No statistically significant difference was found between symptomatic and asymptomatic side. 
TABLE 2: CMAP parameters (patients group and control group).

\begin{tabular}{|c|c|c|c|c|c|c|}
\hline Variable & Group & $N$ & Mean & SD & $5^{\text {th }}$ percentile & $95^{\text {th }}$ percentile \\
\hline \multirow{2}{*}{ Common peroneal nerve distal latency (ms) } & Control & 49 & 4.37 & 0.87 & - & 6 \\
\hline & Patient & 54 & 4.43 & 1.04 & - & - \\
\hline \multirow{2}{*}{ Common peroneal nerve amplitude (mv) } & Control & 49 & 4.34 & 1.69 & 1.6 & - \\
\hline & Patient & 54 & 3.77 & 1.69 & - & - \\
\hline \multirow{2}{*}{ Common peroneal nerve $\mathrm{CV}(\mathrm{m} / \mathrm{s})$} & Control & 49 & 49.57 & 6.25 & 40.6 & - \\
\hline & Patient & 54 & 47.38 & 4.16 & - & - \\
\hline \multirow{2}{*}{ Tibial nerve distal latency (ms) } & Control & 50 & 3.71 & 0.71 & - & 4.7 \\
\hline & Patient & 53 & 3.99 & 0.80 & - & - \\
\hline \multirow{2}{*}{ Tibial nerve amplitude (mv) } & Control & 50 & 11.15 & 4.3 & 3.99 & - \\
\hline & Patient & 53 & 9.2 & 3.38 & - & - \\
\hline \multirow{2}{*}{ Tibial nerve CV (m/s) } & Control & 50 & 45.13 & 5.02 & 38 & - \\
\hline & Patient & 53 & 44.2 & 5.32 & - & - \\
\hline
\end{tabular}

TABle 3: F-wave latencies of the tibial nerve (patients group vs control group).

\begin{tabular}{|c|c|c|c|c|c|}
\hline Variable & Group & $N$ & Mean & $\mathrm{Sd}$ & $P$ value \\
\hline \multirow{2}{*}{ Tibial nerve F-wave minimum latency (ms) } & Control & 50 & 43.42 & 8.88 & \multirow{2}{*}{0.019} \\
\hline & Patient & 54 & 47.42 & 8.86 & \\
\hline \multirow{2}{*}{ Tibial nerve F-wave maximum latency (ms) } & Control & 50 & 52.69 & 6.32 & \multirow{2}{*}{0.005} \\
\hline & Patient & 54 & 56.21 & 6.22 & \\
\hline \multirow{2}{*}{ Tibial nerve F-wave mean latency (ms) } & Control & 50 & 48.71 & 6.27 & \multirow{2}{*}{0.014} \\
\hline & Patient & 54 & 51.73 & 6.68 & \\
\hline
\end{tabular}

TABLE 4: Tibial nerve F-max (control vs symptomatic side and control vs asymptomatic side).

\begin{tabular}{|c|c|c|c|c|c|}
\hline Variable & Population & $N$ & Mean & Std. deviation & $P$ value \\
\hline \multirow{3}{*}{ Tibial nerve F-max latency (ms) } & Control & 50 & 52.69 & 6.31 & - \\
\hline & Patient symptomatic side & 27 & 56.14 & 6.95 & 0.03 \\
\hline & Patient asymptomatic side & 27 & 56.42 & 5.71 & 0.012 \\
\hline
\end{tabular}

H-reflex latencies obtained were compared with values adjusted for age and height [17]. Recorded H-reflex latencies were prolonged in $53.7 \%$ of the 54 studied tibial nerves. $40.7 \%$ of the symptomatic sides showed prolonged H-reflex latency. $29.6 \%$ of the asymptomatic sides showed prolonged H-reflex latency. There was a high statistically significant difference between patients and control in H-reflex latency $(P$ value $=0.001)$ (Table 5$)$. Both symptomatic and asymptomatic sides showed a statistically significant difference when compared to the control $(P$ value $=0.005$ for both) (Table 6). Interside differences of more than $1.5 \mathrm{~ms}$ were obtained in $83.3 \%$ of patients with abnormal H-reflex latency, but no statistically significant difference was found between symptomatic and asymptomatic sides.

Of the 27 of the studied patients, $21(77,8 \%)$ showed abnormal physical examination; 14 of them (66.7\%) showed abnormal nerve conduction studies findings. Of the 6 patients with a normal physical examination, 4 (66.7\%) showed abnormal NCS. Both subjects with chronic symptoms (duration more than 6 months) and those being symptomatic for less than 6 months revealed the same percentage of having abnormal NCS (66.7\% for both groups). 50\% of patients with S1 distribution of symptoms had prolonged H-reflex latency compared to $30 \%$ of patients with L5 dermatomal distribution.
Ankle jerk was absent in 8 patients and prolonged H-reflex latency was found in $62.5 \%$ of them. The percentage of patients with hypoesthesia who had abnormal nerve conduction studies findings was $60 \%$. The percentage of patients with positive SLR tests who had abnormal nerve conduction studies findings was $68.8 \%$.

No statistically significant association was found between abnormal physical examination findings (abnormal gait, absent ankle jerk, hypoesthesia, or positive SLR) and the abnormal nerve conduction studies parameters (reduced amplitude or CV of CMAP, prolonged F-wave latency, or prolonged H-reflex latency) (Table 7). When the association was tested between individual physical examination and nerve conduction studies, it revealed no statistically significant association. Neither absent ankle jerk nor positive straight leg raise test showed statistically significant association with prolonged H-reflex latency (Table 8 and Table 9, respectively).

\section{Discussion}

Nerve conduction studies are considered part of the clinical evaluation of patients with neuromuscular complaints. We conducted our study to define abnormalities in CMAP and late responses ( $\mathrm{F}$-wave and $\mathrm{H}$-reflex) latencies in patients with clinical radiculopathy due to L4/L5 or L5/S1 disc 
TABLE 5: H-reflex latency (patients group vs control group).

\begin{tabular}{lllccc}
\hline Variable & Group & N & Mean & Std. deviation & $P$ value \\
\hline \multirow{2}{*}{ Tibial nerve H-reflex latency $(\mathrm{ms})$} & Control & 50 & 31.09 & 3.25 & $\mathbf{0}$ \\
& Patient & 54 & 33.42 & $\mathbf{0 . 0 0 1}$ \\
\hline
\end{tabular}

TABLE 6: H-reflex latency (control vs symptomatic side and control vs asymptomatic side).

\begin{tabular}{lccccc}
\hline Variable & Population & N & Mean & Std. deviation & $P$ value \\
\hline \multirow{3}{*}{ Tibial nerve H-reflex latency $(\mathrm{ms})$} & Control & 50 & 31.09 & 3.25 & - \\
& Patient symptomatic side & 27 & 33.47 & 3.81 & 0.005 \\
& Patient asymptomatic side & 27 & 33.37 & 3.34 & 0.005 \\
\hline
\end{tabular}

TABLE 7: Association between abnormal clinical examination and abnormal NCS.

\begin{tabular}{lccc}
\hline \multicolumn{1}{c}{ NCS } & Normal & Abnormal & $P$ value \\
Clinical examination & & 4 & 1.000 \\
\hline Normal & 2 & 14 & \\
\hline
\end{tabular}

TABLE 8: Association between abnormal ankle jerk and abnormal H-reflex.

\begin{tabular}{lccc}
\hline $\begin{array}{c}\text { H-reflex } \\
\text { Ankle jerk }\end{array}$ & Normal & Abnormal & $P$ value \\
\hline Normal & 12 & 7 & 0.398 \\
Abnormal & 3 & 5 & \\
\hline
\end{tabular}

TABLE 9: Association between abnormal SLR and abnormal Hreflex.

\begin{tabular}{lccc}
\hline $\begin{array}{l}\text { H-reflex } \\
\text { SLR }\end{array}$ & Normal & Abnormal & $P$ value \\
\hline Normal & 5 & 6 & 0.452 \\
Abnormal & 10 & 16 & \\
\hline
\end{tabular}

herniation and, in addition, to find the association between clinical manifestations and the abnormal nerve conduction study findings.

Our study showed no significant difference in the CMAP parameters of both common peroneal and tibial nerves between patients and the control group. This finding goes with the findings of many previous studies $[5,8,18]$ and contradicts other studies $[6,12]$. In a study conducted by Matsui et al. [6], patients with different grades of motor weakness are included; their results showed a decrease in CMAP amplitude and prolonged latency that correlate with the degree of motor weakness [6], while in our study none of the patients suffered from any degree of motor weakness; this might explain the lack of the significant difference between the control and the patient group. Nafissi et al. reported significant abnormality in CMAP amplitude of peroneal nerve in patients with pretibial muscle weakness [12].

In the current study, F-wave of both common peroneal and tibial nerves was obtained, and minimum, maximum, and mean latencies were evaluated. There was a statistically significant difference between patients and normal subjects in the tibial F-wave minimum, maximum, and mean latencies, which coincide with previous studies $[5,11]$. The maximum latency was the most significant of the three. When compared with control, both symptomatic and asymptomatic sides showed a statistically significant difference in the maximum latency. A study conducted by Toyokura and Murakami in 1996 reported high sensitivity (70\%) of F-minimum, F-maximum, F-difference, and F-duration on the affected side [5]. Toyokura et al. carried out another study in 2002; they studied F-minimum and F-duration of tibial nerve in mild $\mathrm{S} 1$ radiculopathy. They concluded that F-minimum and F-duration corrected by the subject's height $(\mathrm{F}-\mathrm{min} / \mathrm{H})$ were both significantly longer on the affected side than on the unaffected side and longer than the normal group. However, the incidence of abnormality was very low for both parameters [8].

Our study showed no statistically significant difference between controls and patients in peroneal F-wave latencies, contrary to a study carried out by Weber, who concluded from his study that tibial nerve F-wave is not sensitive and more cases of $\mathrm{S} 1$ radiculopathy can be detected by $\mathrm{F}$-wave of the common peroneal nerve [18]. Their findings might be explained by the fact that most of their patients had L5 radiculopathy or combined L5 and S1 radiculopathy, while our study included more cases with S1 radiculopathy than L5 radiculopathy $(59.26 \%$ and $37.04 \%$, respectively).

Significant prolongation of H-reflex latency was obtained in this study and the percentage of tibial nerves that showed prolonged H-reflex was more on the symptomatic side; this was consistent with previous studies [7, 9, 10, 19]. Both symptomatic and asymptomatic sides revealed significant prolongation in latency when compared to the control group. This could be explained by the fact that disc prolapse tends to affect both nerve roots though one side remains subclinical. In contrast to the results of Haroun et al. and Alrowayeh et al., who studied H-reflex in S1 radiculopathy, our study result failed to show a statistically significant difference between symptomatic and asymptomatic sides [7, 10].

From patients with abnormal ankle jerk, 62.5\% showed $\mathrm{H}$-reflex latency prolongation in our study. This was lower than what was obtained by Haroun et al. in their study. They reported that all their patients with abnormal ankle jerk showed prolonged H-reflex latency [7]. Our study failed to show a statistically significant association between duration of symptoms and abnormal NCS in contrast to a previous study that reported a significant relationship between the duration of clinical symptoms and the electrophysiological findings [12]. 
Our current study showed no statistically significant association between physical examination and nerve conduction studies. Therefore, the physical examination is not an adequate predictor of abnormal nerve conduction studies in subjects with symptoms suggesting radiculopathy. This coincides with the conclusion of many previous studies [12-15]. Lauder et al. concluded that having at least one abnormal physical examination finding makes the probability of having an abnormal electrodiagnostic study more likely. However, having normal physical examination does not rule out the probability of finding abnormal EDX. They concluded also that history and clinical examination are not a perfect tool in diagnosing radiculopathy or predicting electrodiagnostic study but remain an essential part of clinical evaluation of patients to aid in formulating a differential diagnosis and guiding the electrodiagnostic study [14]. Inal et al. also emphasized that neurological examination and the electrodiagnostic test must be used and interpreted jointly [15]. Finally, NCS can be used to aid in the diagnosis when clinical findings do not coincide with MRI findings (e.g., non-compressive radiculopathy) [20], or when it is difficult to obtain MRI (e.g., a patient who has MRI-incompatible spinal cord stimulator).

\section{Conclusion}

We concluded from our findings that NCS is a valuable tool in evaluating subjects with lumbosacral radiculopathy, $\mathrm{H}$-reflex latency prolongation being the commonest encountered abnormality in our study. Also, we concluded that normal physical examination was frequently encountered with abnormal NCS as well as abnormal physical examination detected in patients with normal NCS findings. Therefore, both of them should be evaluated simultaneously in patients with history suggesting radiculopathy.

\section{Data Availability}

Data are available from the corresponding author upon reasonable request.

\section{Conflicts of Interest}

The authors report no conflicts of interest.

\section{References}

[1] S. T. Canale, J. H. Beaty, Campbell's Operative Orthopaedics, Elsevier, Amsterdam, Netherlands, 12th edition, 2013.

[2] D. Moore, "Lumbar disc herniation," 2015, http://www. orthobullets.com/spine/2035/lumbar-disc-herniation.

[3] D. S. Kreiner, S. W. Hwang, J. E. Easa et al., "An evidencebased clinical guideline for the diagnosis and treatment of lumbar disc herniation with radiculopathy," The Spine Journal, vol. 14, no. 1, pp. 180-191, 2014.

[4] R. I. Chichkova and L. Katzin, "EMG and nerve conduction studies in clinical practice," 2010, http://practicalneurology. com/2010/02/emg-and-nerve-conduction-studies-in-clinicalpractice.

[5] M. Toyokura and K. Murakami, "F-wave study in patients with lumbosacral radiculopathies," Electromyography and Clinical Neurophysiology, vol. 37, no. 1, pp. 19-26, 1996.
[6] H. Matsui, M. Kanamori, Y. Kawaguchi, H. Kitagawa,H. Nakamura, and H. Tsuji, "Clinical and electrophysiologiccharacteristics of compressed lumbar nerve roots," Spine, vol. 22, no. 18, pp. 2100-2105, 1997.

[7] N. S. Haroun, N. M. Sherief, M. M. Abdul-Hakiem, A. M. AlGanzoury, A. M. K. Al-Gogary, and M. R. S. Al-Helowe, "Hreflex in the diagnosis of lumbosacral radiculopathy," Egyptian Rheumatology and Rehabilitation, vol. 26, no. 4, pp. 763-769, 1999.

[8] M. Toyokura and T. Furukawa, "F wave duration in mild S1 radiculopathy: comparison between the affected and unaffected sides," Clinical Neurophysiology, vol. 113, no. 8, pp. 1231-1235, 2002.

[9] S. C. Cho, M. A. Ferrante, K. H. Levin, R. L. Harmon, and Y. T. So, "Utility of electrodiagnostic testing in evaluating patients with lumbosacral radiculopathy: an evidence-based review," Muscle \& Nerve, vol. 42, no. 2, pp. 276-282, 2010.

[10] H. N. Alrowayeh and M. A. Sabbahi, "H-reflex amplitude asymmetry is an earlier sign of nerve root involvement than latency in patients with S1 radiculopathy," BMC Research Notes, vol. 4, no. 1, p. 102, 2011.

[11] M. Gencer, K. Uluc, Y. Cetinkaya et al., "Clinical utility of F wave parameters in unilateral S1 radiculopathy," Neurosciences (Riyadh, Saudi Arabia), vol. 16, no. 3, pp. 237-241, 2011.

[12] S. Nafissi, S. Niknam, and S. S. Hosseini, "Electrophysiological evaluation in lumbosacral radiculopathy," Iranian Journal of Neurology, vol. 11, no. 3, pp. 83-86, 2012.

[13] T. D. Lauder, T. R. Dillingham, M. Andary et al., "Effect of history and exam in predicting electrodiagnostic outcome among patients with suspected lumbosacral radiculopathy," American Journal of Physical Medicine \& Rehabilitation, vol. 79, no. 1, pp. 60-68, 2000.

[14] T. D. Lauder, "Physical examination signs, clinical symptoms, and their relationship to electrodiagnostic findings and the presence of radiculopathy," Physical Medicine and Rehabilitation Clinics of North America, vol. 13, no. 3, pp. 451-467, 2002.

[15] E. E. İnal, F. Eser, L. A. Aktekin, E. Öksüz, and H. Bodur, "Comparison of clinical and electrophysiological findings in patients with suspected radiculopathies," Journal of Back and Musculoskeletal Rehabilitation, vol. 26, no. 2, pp. 169-173, 2013.

[16] S. Yousif, A. Musa, A. Ahmed, and A. Abdelhai, "Correlation between findings in physical examination, magnetic resonance imaging, and nerve conduction studies in lumbosacral radiculopathy caused by lumbar intervertebral disc herniation," Advances in Orthopedics, vol. 2020, Article ID 9719813, 6 pages, 2020.

[17] R. Buschbach and N. Prahlow, Manual of Nerve Conduction Studies, Demos Medical Publishing, New York, NY, United States, 2nd edition, 2006.

[18] F. Weber, "The diagnostic sensitivity of different F wave parameters," Journal of Neurology, Neurosurgery \& Psychiatry, vol. 65, no. 4, pp. 535-540, 1998.

[19] M. Wojtysiak, J. Huber, A. Wiertel-Krawczuk, A. Szymankiewicz-Szukała, J. Moskal, and J. Janicki, "Pre-and postoperative evaluation of patients with lumbosacral disc herniation by neurophysiological and clinical assessment," Spine, vol. 39, no. 21, pp. 1792-1800, 2014.

[20] M. J. Albeck, G. Taher, M. Lauritzen, and W. Trojaborg, "Diagnostic value of electrophysiological tests in patients with sciatica," Acta Neurologica Scandinavica, vol. 101, no. 4, pp. $249-254,2000$. 